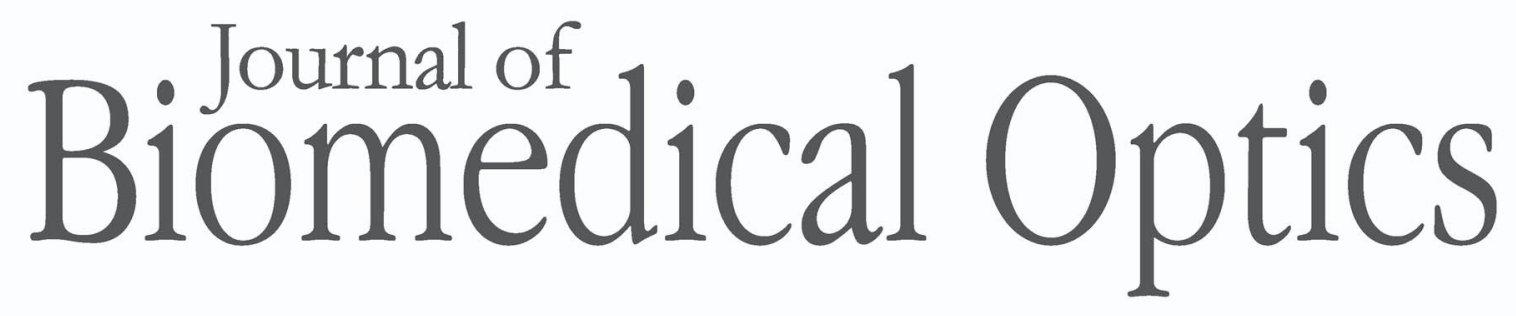

\title{
Recognition of serous ovarian tumors in human samples by multimodal nonlinear optical microscopy
}

Javier Adur

Vitor B. Pelegati

Leverson F. L. Costa

Luciana Pietro

Andre A. de Thomaz

Diogo B. Almeida

Fatima Bottcher-Luiz

Liliana A. L. A. Andrade

Carlos L. Cesar 


\title{
Recognition of serous ovarian tumors in human samples by multimodal nonlinear optical microscopy
}

\author{
Javier Adur, ${ }^{\mathrm{a}, \mathrm{b}}$ Vitor B. Pelegati, ${ }^{\mathrm{a}}$ Leverson F. L. Costa, ${ }^{\mathrm{a}}$ Luciana Pietro, $^{\mathrm{c}}$ Andre A. de Thomaz, ${ }^{\mathrm{a}}$ \\ Diogo B. Almeida, ${ }^{a}$ Fatima Bottcher-Luiz, ${ }^{d}$ Liliana A. L. A. Andrade, ${ }^{c}$ and Carlos L. Cesar ${ }^{a}$ \\ a University of Campinas (UNICAMP), Biomedical Lasers Application Laboratory, Research Center in Optics \\ and Photonics, "Gleb Wataghin" Physics Institute, Campinas, San Pablo, 13083-859 Brazil \\ ${ }^{b}$ National University of Entre Ríos (UNER), Microscopy Laboratory Applied to Molecular and Cellular Studies, School \\ of Bioengineering, Entre Rios, Oro Verde 3101, Argentina \\ 'University of Campinas (UNICAMP), Department of Pathology, Campinas, San Pablo, 13083-859 Brazil \\ dUniversity of Campinas (UNICAMP), Department of Obstetrics and Gynecology, Campinas, San Pablo, \\ 13083-859 Brazil
}

\begin{abstract}
We used a multimodal nonlinear optics microscopy, specifically two-photon excited fluorescence (TPEF), second and third harmonic generation (SHG/THG) microscopies, to observe pathological conditions of ovarian tissues obtained from human samples. We show that strong TPEF + SHG + THG signals can be obtained in fixed samples stained with hematoxylin and eosin $(\mathrm{H} \& \mathrm{E})$ stored for a very long time, and that $\mathrm{H} \& \mathrm{E}$ staining enhanced the THG signal. We then used the multimodal TPEF-SHG-THG microscopies in a stored file of H\&E stained samples of human ovarian cancer to obtain complementary information about the epithelium/stromal interface, such as the transformation of epithelium surface (THG) and the overall fibrillary tissue architecture (SHG). This multicontrast nonlinear optics microscopy is able to not only differentiate between cancerous and healthy tissue, but can also distinguish between normal, benign, borderline, and malignant specimens according to their collagen disposition and compression levels within the extracellular matrix. The dimensions of the layers of epithelia can also be measured precisely and automatically. Our data demonstrate that optical techniques can detect pathological changes associated with ovarian cancer. ( 2011 Society of Photo-Optical Instrumentation Engineers (SPIE). [DOI: 10.1117/1.3626575]
\end{abstract}

\begin{abstract}
Keywords: third harmonic generation microscopy; second harmonic generation microscopy; serous ovarian cancer; extracellular matrix; collagen; nonlinear image processing.

Paper 11145RR received Mar. 22, 2011; revised manuscript received Jul. 28, 2011; accepted for publication Aug. 1, 2011; published online Sep. 14, 2011.
\end{abstract}

\section{Introduction}

Any new microscopy, such as nonlinear optics microscopy (NLO) technique images, should be compared to hematoxylin and eosin (H\&E) stained images not only to make sure of the interpretation of the image, but also to highlight the differences and to establish the strengths and weaknesses of each technique. Therefore, the learning curve of the new microscopy images involves a comparison against the established technique.

One very important reason for using NLO with H\&E fixed samples is that long time-scale processes in the range of years (such as cancer processes), can be followed using an already built library of H\&E stained slides of cancer samples stored around the world. If a new microscopy technique can see new features on those stored materials then it would be possible to make a retrospective study of long time-scale biological processes.

NLO microscopy started with two photon excited fluorescence (TPEF), or multiphoton, microscopy, ${ }^{1}$ which has very few advantages for standard pathology procedures. However, if one already has a TPEF experimental setup, second harmonic generation (SHG) and third harmonic generation (THG) microscopy can be obtained without much effort and cost, simply by adding extra nondescanned detectors and bandpass optical

Address all correspondence to: Javier Adur, National University of Entre Rios, Departamento Biologia, Ruta 11 Km10, Oro Verde 3101, Entre Rios Argentina; Tel: 0054-343-4975100; E-mail: jadur@bioingenieria.edu.ar. filters. ${ }^{2,3}$ SHG visualizes the collagen assembly directly, which is very important for understanding the progress and metastasis of cancer. ${ }^{4-8}$ SHG does not present photobleaching and can provide information about the dimensions and directionality of fibrils. Therefore, reanalyzing old H\&E samples with SHG microscopy would not destroy the register and can reveal unseen features, making it worth using in pathology slide files. On the other hand, the THG signal is enhanced for interfaces and optical nonhomogeneities. Besides these structural factors, THG is sensitive to local differences in third-order nonlinear susceptibility, refractive index, and dispersion. It has recently been demonstrated that THG microscopy can highlight the nuclei within thick tissue and can be combined efficiently with multiphoton fluorescence and SHG microscopy. ${ }^{9}$ Being able to see and count the nuclei is very important for pathology diagnoses. ${ }^{10}$ Fortunately, the H\&E procedure can actually enhance the THG signal and can be observed even in stored samples that are more than 10 years old. ${ }^{11}$

The purpose of this paper is to show how the combined use of these three techniques is very valuable for cancer studies, revealing valuable and complementary information about the tissue under investigation. We apply NLO microscopy techniques to study H\&E stained slides from epithelial ovarian cancer biopsies of different patients, stored in our "library" for up to 15 years.

1083-3668/2011/16(9)/096017/10/\$25.00 @ 2011 SPIE 
This entity is responsible for the highest mortality among gynecologic cancers. ${ }^{12}$ As a disease characterized by nonspecific symptoms, epithelial ovarian cancer is typically diagnosed in the late stage. ${ }^{13,14}$ It is therefore imperative to better understand the fundamental changes in ovarian carcinogenesis so as to develop early diagnostic measures that could improve the outcome of the disease.

In this study, we assess the applicability of the multimodal nonlinear optical microscopy approach to the histopathological evaluation of serous ovarian neoplasms (the most common type) from human biopsies whose sections were $H \& E$ stained. Optical biomarkers of ovarian cancer underwent specific prior evaluation with nonlinear microscopy. ${ }^{15}$ However, few studies have been reported combining all techniques in the same complete ovarian cancer study. ${ }^{15-17}$ Our principal aim was to investigate SHG-specific features of the collagen structure that were potentially associated with morphological alterations in the benign, borderline, and malignant samples. The study provides the basis for future applications of nonlinear multicontrast microscopy for histological investigations and in particular for cancer imaging and ovarian carcinoma diagnosis.

\section{Methods}

\subsection{Experimental Setup}

Figure 1 shows the experimental setup, consisting of an inverted microscope IX-81, equipped with a FV300 scanner and the laser combiner FV-5 COMB2 by Olympus (Olympus, Tokyo, Japan). A computer controlled XY stage ProScan (Prior Scientific) allowed sample positioning perpendicular to the optical axis. All images were acquired with a PLANAPO 40X, N.A. 1.3 oil immersion objective (Olympus, Tokyo, Japan).
The two photon excitation, SHG and THG, were excited with a Ti:Sapphire Mai Tai HP Spectra-Physics (Irvine, USA) which provides $100 \mathrm{fs}$ pulses from 690 to $1040 \mathrm{~nm}$ with a repetition rate of $80 \mathrm{MHz}$ and powers from 1 to $3.5 \mathrm{~W}$. It was equipped with a DeepSee for group velocity dispersion compensation and a broadband half wave plate (HWP), coupled to a calcite polarizing beam splitter (PBS), as an attenuator. The beam was coupled to the scan head through a custom made port and dichroic after a collimating telescope (L1, L2) used to adjust both the beam diameter to fill the objective back-aperture and the beam focus position on the microscope focal plane.

All signals, TPEF, SHG and THG, were detected with R3896 photomultiplier tubes (PMTs) (Hamamatsu Photonics, Hamamatsu City, Japan). The TPEF was detected with the internal scan head PMT after a blocking filter (SP) E-700-SP (Omega Filters) to prevent back-reflected laser light with a completely open pinhole. Only transmitted SHG and THG signals were collected. To avoid THG UV light blocking by the optical elements of the microscope we built a special PMT support to hold it as close to the sample as possible, without touching it, but with enough space for the optical filters. The large area of the PMT assured a good numerical aperture collection angle. To detect an SHG signal we used an E700-SP short pass filter followed by a narrow (10 nm FWHM) bandpass optical filter centered at half the excitation wavelength (475BP, Bio-Rad) to reject the excitation laser beam and any unwanted fluorescence. To collect THG images we replaced both filters with two colored glass filters U-340 $\pm 30 \mathrm{~nm}$ (Hoya Corporation). SHG and THG images were acquired one after the other due to the exchange of the optical filters, but simultaneously with the TPEF images. Both TPEF were compared to make sure that the sample did not move during the optical filter exchange procedure.
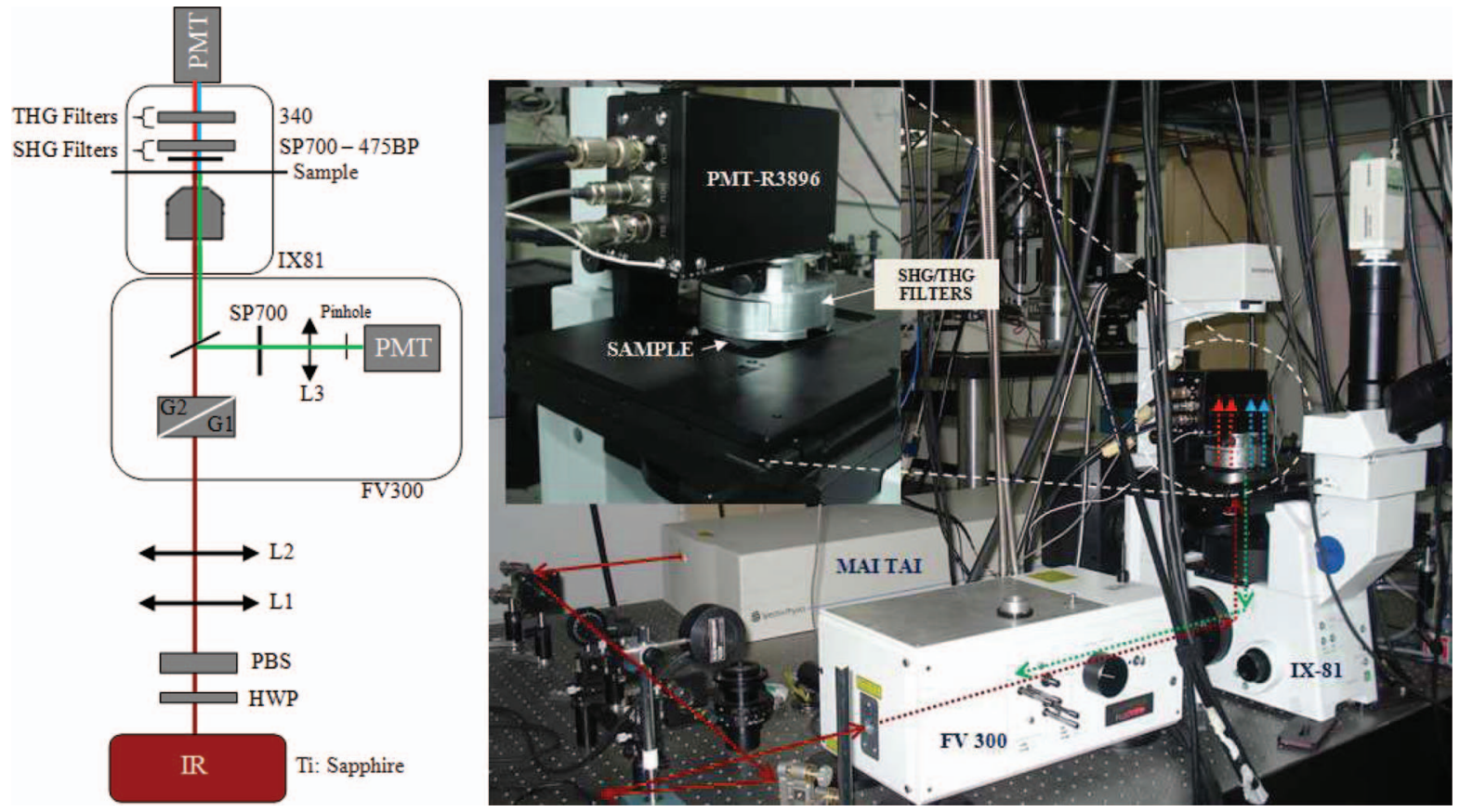

Fig. 1 Experimental setup to TPEF, SHG, and THG microscopy. Left figure shows a simplified optical scheme. HWP: half wave plate, PBS: polarizing beam splitter, L1-L2: telescope lens, G1-G2: galvanometer mirrors, L3: collecting lens, PMT: photomultiplier tubes, BP: bandpass filter, SP: short pass filter. Right figure shows real setup based on an inverted microscope Olympus IX-81and an Olympus FV300 confocal scanning head (Olympus, Japan). The SHG (red lines) and THG (blue lines) are collected in a transmitted light configuration. The TPEF (green lines) is collected in backscattering configuration. 


\subsection{Human Materials, Sample Preparation, and Histopathology Analysis Criteria}

Tissues were obtained from women attending a Women's Health Center CAISM (from 1994 to 2009), Campinas, SP, Brazil and the project was approved by the Institutional Ethics Committee (Faculty of Medical Sciences, Unicamp). Ovarian specimens collected during surgical procedures were prepared following a standard histological procedure: fixed in $10 \%$ buffered formalin, embedded in paraffin, cut in $4-\mu \mathrm{m}$ thick sections and mounted on slides. Slides from serial sections were stained with $H \& E$ and covered with coverslips. We also used some unstained slides to compare with $H \& E$ stained slides. Each $H \& E$ stained tissue section was evaluated by a certified pathologist, based on established World Health Organization histological criteria. ${ }^{18}$ The histological diagnosis was determined from evaluation of multiple sections from each specimen. All histological sections were reviewed by the same pathologist to avoid bias in the biopsy analysis as well as to promote a homogeneously histological classification of the tumors. A total of 20 ovarian specimens (obtained from different patients with ages ranging from 40 to 75 years) were analyzed in this study and classified as normal ovarian tissue ( 5 cases) or ovarian serous tumors ( 3 adenomas, 2 borderline type, and 10 adenocarcinomas). For qualitative and quantitative analysis of the stromal and epithelial structures, 3 images were selected and collected in each case, resulting in a total of 60 images from all 20 tissue samples.

\subsection{Image Acquisition and Analysis}

After some experimentation we observed that a $940 \mathrm{~nm}$ laser beam could excite the three signals, TPEF, SHG, and THG, at the same time. We therefore decided that all images would be acquired with the $940 \mathrm{~nm}$ excitation, generating an SHG at $470 \mathrm{~nm}$ and a THG at $313 \mathrm{~nm}$ with an average laser power of $20 \mathrm{~mW}$ in the sample. This power level was sufficiently low to avoid photobleaching and photodamages. All images were acquired with $512 \times 512$ pixel spatial resolution, using a pixel dwell time of $5 \mathrm{~ms}$, with total scanning time of order of $3 \mathrm{~s}$, after a 5 frames Kalman filtering. A digital camera placed on the microscope viewfinder was used to obtain usual wide field $\mathrm{H} \& \mathrm{E}$ images with white light illumination.

Images (pseudo-green TPEF, pseudo-red SHG, and pseudomagenta THG) were combined into a single image for visualization using Olympus Fluoview Image Browser. For visual presentation, images were optimized by background thresholding and histogram stretching. Although these techniques improved visual image contrast and presentation, all quantitative image analyses processed with ImageJ $(\mathrm{NIH}$, available from http://rsb.info.nih.gov/ij) were performed on the unprocessed images to avoid any artifacts.

Figure 2 shows a diagram of the different steps followed to obtain measurements from THG and SHG images. Based on THG images, the width of epithelium [Fig. 2(c)] and the number of nuclei were calculated [Fig. 2(d)]. The epithelial layers were determined from three regions (top, center, and bottom) of the same image between the epithelial surface and the epithelial/stromal interface [Fig. 2(c)] (at which collagen was first identified and/or the cellular fluorescence was no longer present). The average of these regions was used as the final

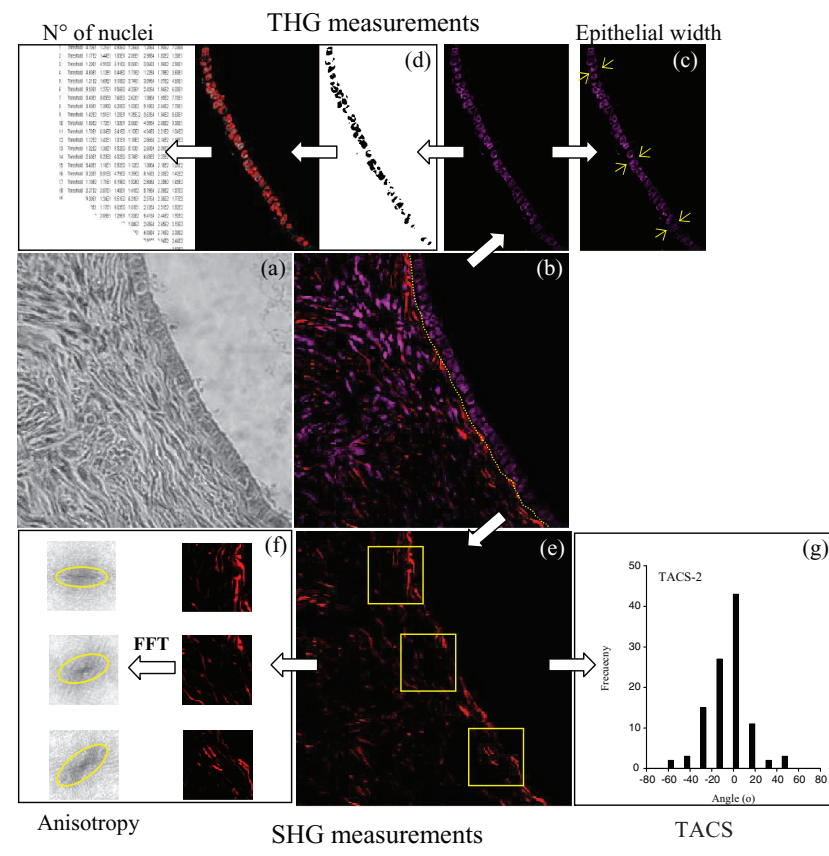

Fig. 2 Diagram of the steps involved in the measurements of THG and SHG signals. (a) Classic H\&E-stained signal used as reference. (b) Combination of SHG (red) + THG (magenta) signal captured from (a), epithelial/stromal interface is indicated (yellow outline). From the THG image, epithelial width [from three regions: see arrowhead (c)] and the number of nuclei (d) was calculated with Imagej software. From the SGH image, three different regions near the epithelial/stromal interface (e) were used to calculate the FFT transforms ( $f$ ) and fit to ellipse to estimate the anisotropy. A TACS was done measuring the collagen fiber angle relative to the epithelium (g).

width. The number of nuclei was counted in the epithelial layers using "Particle Analysis-Nucleus Counter" plug-ins of ImageJ. This plug-in performs a threshold automatically and presents the results in a table where each nucleus can be identified [Fig. 2(d)]. The accuracy of this technique was verified by comparing it to the number of nuclei counted manually from two images from normal biopsies.

For collagen assessment based on SHG [Fig. 2(e)], we calculated the anisotropy [Fig. 2(f)] and tumor-associated collagen signatures (TACS) [Fig. 2(g)] of the SHG images. ${ }^{6}$ The analysis of the anisotropy was performed by means of the twodimensional discrete Fourier transform (2D DFT) computed using the FFT of ImageJ. For calculations, three regions in the SHG images were selected near the epithelial surface [Fig. 2(e)]. The frequency distribution in a Fourier-transformed image can be analyzed to retrieve the pattern of image line directions, so as to characterize the geometry of the image texture. Thus, the FFT of each region was computed and the logarithm of the absolute value of the calculated FFT was displayed. Next, the FFT magnitude was threshold to levels 0 or 1 . The degree of FFT deformation was quantitatively calculated by fitting the result with an ellipse and by calculating the ratio between its short and long axes, i.e., its aspect ratio (AR). AR, ranging from 0 to 1 , can give a measure of the anisotropy of the sample. The sample is more anisotropic as the AR is close to 0 , whereas it is more isotropic when the AR is close to 1. For TACS, the collagen fiber angle relative to the tumor was quantified. The tumor boundary was 
defined and the angle relative to the tangent of tumor boundary was measured [Fig. 2(g)].

Because all data come from a digital analysis of the images and all the processing was done automatically, a blind analysis was not necessary.

\subsection{Statistical Analysis of Morphologic Features}

Differences in morphologic features of subsample images were analyzed statistically by the analysis of variance (ANOVA) test with Tukey-Kramer multiple comparisons test. Subsamples are the three regions imaged within a tissue sample. If significant subsample variability was found in these features, then the features obtained from the subsamples were analyzed separately. Otherwise, the features obtained from the subsamples were averaged for further analysis. The aspect ratio between normal tissues and each histological classification of ovarian cancer, and the amount of collagen fibers at different angles with respect to epithelia were compared by a $t$-test. Data were analyzed with SPSS 10.0 software.

\section{Results and Discussions}

\subsection{Multimodal Nonlinear Microscopy of H\&E Stained Samples Reveals Structural Organization of the Ovary}

As a first step and to compare the effects of the H\&E classical stained method in nonlinear microscopy assays, two types of tissue sections from normal ovarian biopsies were prepared: unstained slides and H\&E-stained slides. Samples were sectioned from the same tissue block and captured under the same powerfield and magnification conditions. Figure 3 shows a comparison between TPEF, SHG, and THG images of H\&E stained and unstained samples. It is clear that TPEF and THG images are much brighter in the H\&E samples, while SHG is about the same. Figure 3(c) demonstrates that multimodal NLO microscopy provides high quality images in $\mathrm{H} \& \mathrm{E}$ stained samples.

Although H\&E staining was expected to enhance fluorescence signals, we also observed that staining increased the THG signals arising from endogenous molecules. Nonlinear microscopy has been used to investigate $\mathrm{H} \& \mathrm{E}$ histological sections, ${ }^{19}$ as well as tissue stained separately with Hematoxilin ${ }^{20}$ and Eosin dyes. ${ }^{21}$ These studies demonstrated that using stain-absorbing levels that coincide with the multiphoton frequencies of the excitation light, hematoxylin stain may selectively enhance THG yield at cell nuclei. The absorption spectrum of hematoxylin shows a broad range of absorption at the visible light band, achieving the two- and three-photon resonance enhancement criteria. ${ }^{20}$ On the other hand, it was recently observed that staining the tissue with acidic hematoxiylin solution causes high concentrations of charged hemalum complexes to migrate to the compartments with negatively charged DNA and RNA via Coulomb interactions. During the completion of the staining, the $p \mathrm{H}$ becomes basic initiating the conversion of hemalums to the neutral form, $\mathrm{HmAl}^{0}$, and induces

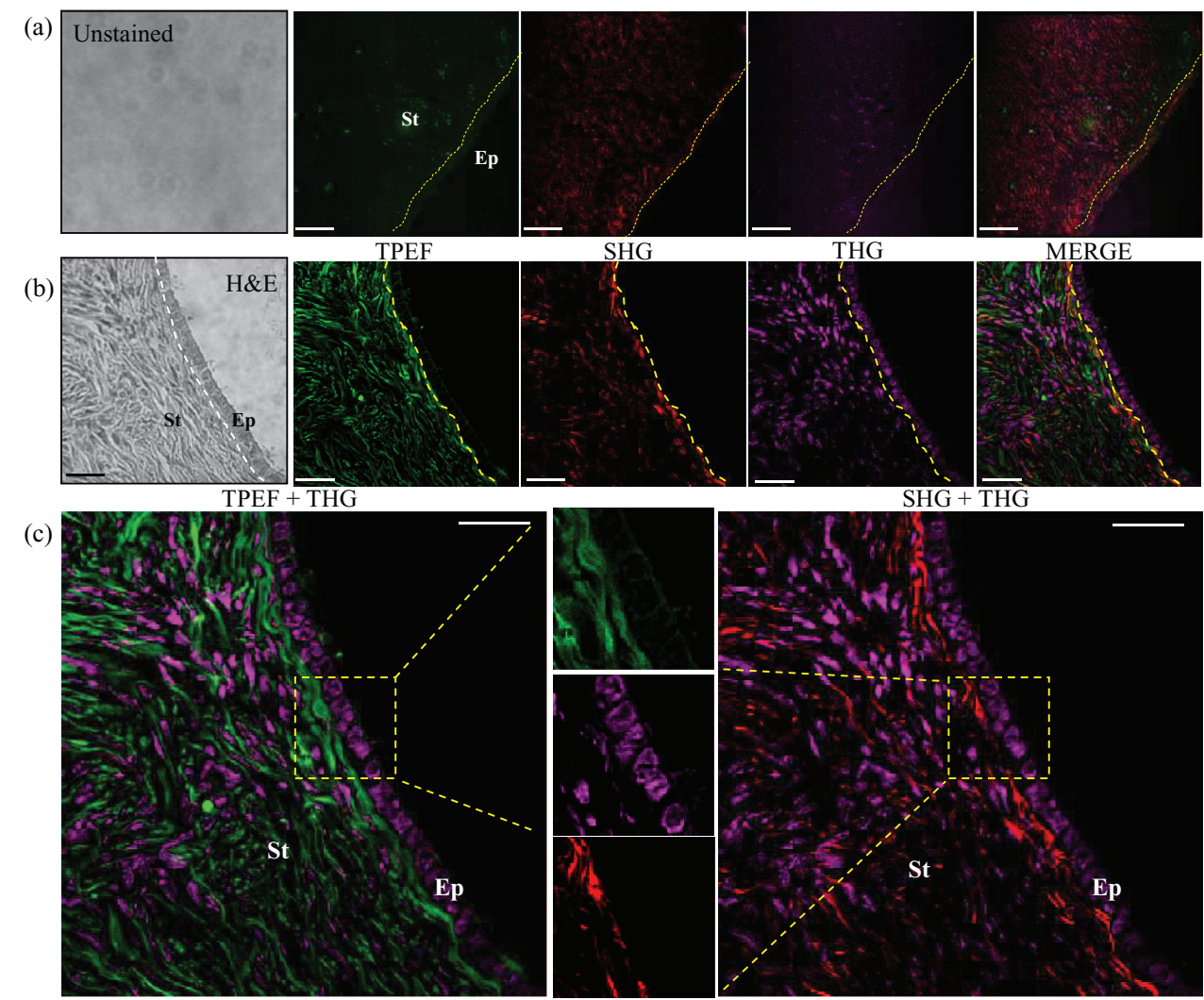

Fig. 3 Multicontrast imaging of unstained (a) and stained normal ovary tissue with H\&E staining (b). Epithelial/stromal interface is indicated (yellow outline). TPEF: two-photon excited fluorescence, SHG: second harmonic generation, THG: third harmonic generation, and MERGE: combination of TPEF + SHG + THG. (c) Enlarged images of (b), the insets shows the principal contrast produced for each technique. All images $(512 \times 512$ pixels $)$ were obtained under the same imaging conditions. The rate of scanning was 3 frames/s, and images were obtained by summing 5 frames. All scale bars are $20 \mu \mathrm{m}$. Ep: epithelium, St: stromal. 
aggregation of the complexes, which exhibit a strong generation of the third harmonic inside the cells. In H\&E stained samples, the twofold enhancement of THG most probably originates due to an interaction between the hemalum complexes and eosin at the surface of $\mathrm{HmAl}^{0}$ precipitates. The THG observed in $\mathrm{H}-$ and $\mathrm{H} \& \mathrm{E}$-stained tissue are stronger than in dried precipitates, most probably due to differences in refractive index and nonlinear susceptibility of the tissue environment, and interaction of the dyes with chromatin. ${ }^{11}$ Our observed enhancement of THG signals images originating almost exclusively from the nuclear regions agrees with these observations.

Moreover, it is known that several endogenous protein structures give rise to SHG signal, ${ }^{22}$ namely: collagen, myosin, and microtubules. In this work we find similar results in the ovary [Fig. 3(c)], where the TPEF signal was weak from the nuclear regions but strong in the regions outside the nucleus. The SHG corresponds to collagen within the stromal connective tissue and the corresponding THG signal highlights the nuclei revealing the locations of the cells within the tissue. The border of the nuclear membrane is clearly visualized in THG with both shape and size of nuclei easily observable [Fig. 3(c), enlarged)].

In summary, comparing NLO with established H\&E-stained techniques we find that new features in the ovarian stroma (orientation and organization of collagen) can be analyzed with SHG imaging. Moreover, the structural information revealed by each nonlinear contrast mechanism can be isolated and analyzed separately, while their superimposition allows a better comparison and understanding of the spatial organization of the tissue. For example, the THG and SHG signals can clearly identify the epithelial/stromal interface, which is very important in analyzing the progression of ovarian cancer.

\subsection{THG-based Morphometric Analysis Allows Detection of Changes during Ovarian Carcinogenesis}

According to previous results, the aim of the second part of the study was to qualitatively and quantitatively determine whether a THG signal from nucleus could resolve epithelial differences in ovarian tumors. Epithelium was easily identified from SHG + THG combination images [Figs. 4(a)-4(d)] and separate with ImageJ software [Figs. 4(e)-4(h)]. Qualitatively we recognized differences in surface epithelium from normal and abnormal ovarian tissues. Morphologically, all normal samples were similar. In the representative normal sample shown in Fig. 4(e), the cells were closely packed and distributed in one layer. This homogeneous size of cells and uniform distribution would be expected in normal ovarian surface epithelium. Samples from serous adenoma show tall ciliated and nonciliated cuboidal cells with elongated nuclei, and also in one cell layer and uniform distribution [Fig. 4(f)]. Unlike normal tissue, in a borderline tumor [Fig. 4(g)] and serous adenocarcinoma [Fig. 4(h)] the epithelial surface showed cells of varying sizes distributed in multiple layers, appearing to clump together, consistent with the histology of neoplastic tissue, including cellular atypia and proliferation [Figs. 4(g), 4(h) and insets].

We also performed an automatic quantitative evaluation of the quantity of nuclei [Fig. 4(i)] and the epithelial width [Fig. 4(j)] to assess epithelial transformations. These values increased from normal tissues to serous adenocarcinoma. How- ever, between normal and serous adenoma samples the differences were not significant for both values. Instead, the number of nuclei in borderline and serous adenocarcinoma lesions (198 \pm 12 and $240 \pm 34$, respectively) was significantly different with respect to normal and benign samples $(42 \pm 10$ and $58 \pm 7$, respectively). These results confirm the observed proliferation. On the other hand, the epithelial stratification was confirmed by measuring the epithelial width. This parameter was $22.47 \pm 6.17$ $\mu \mathrm{m}$ in serous adenocarcinoma and $12.37 \pm 4.28 \mu \mathrm{m}, 11.52$ $\pm 2.04 \mu \mathrm{m}$, and $7.54 \pm 1.98 \mu \mathrm{m}$ in borderline, serous adenoma, and normal samples, respectively.

Our results correlate well with quantifications previously performed on an H\&E stained section from the same histological type tumors. ${ }^{18}$ In summary, using a THG signal in biopsies from patients diagnosed with ovarian cancer, abnormal morphologic changes were observed, consistent with focal or diffuse cellular proliferation and atypia. Therefore, for the first time we demonstrated the usefulness of a THG signal to quantify transformation in surface epithelium of the ovary.

\subsection{Quantitative Differences between Normal and Neoplastic Ovarian Stromal are Resolved with SHG Microscopy}

SHG has already been shown to have potential applicability for cancer diagnosis by revealing changes in the extracellular matrix in tumors relative to normal tissues. For example, some researchers used SHG to identify tumor borders in basal cell carcinoma lesions by imaging the collagen assembly. ${ }^{23,24}$ Similarly, in a mouse model of breast carcinoma, Keely identified distinct stages of invasion by measuring changes of the angle of collagen fibers with respect to tumor boundaries. ${ }^{6,25}$ Jain also demonstrated that the increased collagen concentration (i.e., desmoplasia) associated with a tumor from implanted melanoma cells was measurable by SHG. ${ }^{26}$ However, few studies have exploited this technique for ovarian cancer. Therefore, the focus of the third part of the study was to qualitatively and quantitatively determine whether SHG could discriminate differences between the stroma of normal and tumoral ovarian tissues (serous adenoma, borderline, and serous adenocarcinoma).

The SHG signal is stronger when the long axis of the fibril is parallel to the laser polarization and is minimized when that axis becomes perpendicular to the laser polarization. However, even for 5- $\mu \mathrm{m}$ thick samples one observes that the fibril images never disappear. The intensity ration between the polarization maximum/minimum can be 1.9 for thin nonscattering samples but it falls to 1.2 for thick highly-scattering samples. ${ }^{27}$ Moreover, the optical elements of the microscope introduce a degree of ellipticity in a polarized incident beam of the order of $15 \%$ to $20 \%$, meaning that the polarization at the sample is not linearly polarized, which decreases the maximum/minimum ratio discussed above. This means that the polarization can change the intensity of the SHG signal but not enough to hide images of perpendicular fibrils. Our analysis was made solely on the SHG pattern (directionality of the fibers), never on the SHG intensity. The fact that our samples, although $4 \mu \mathrm{m}$ thick, were highly scattering because of the H\&E fixation procedure, helped to see fibrils in all directions without much loss in intensity.

Figure 5 shows representative H\&E-stained and SHG images of tissues diagnosed as normal ovary [Fig. 5(a)], serous 

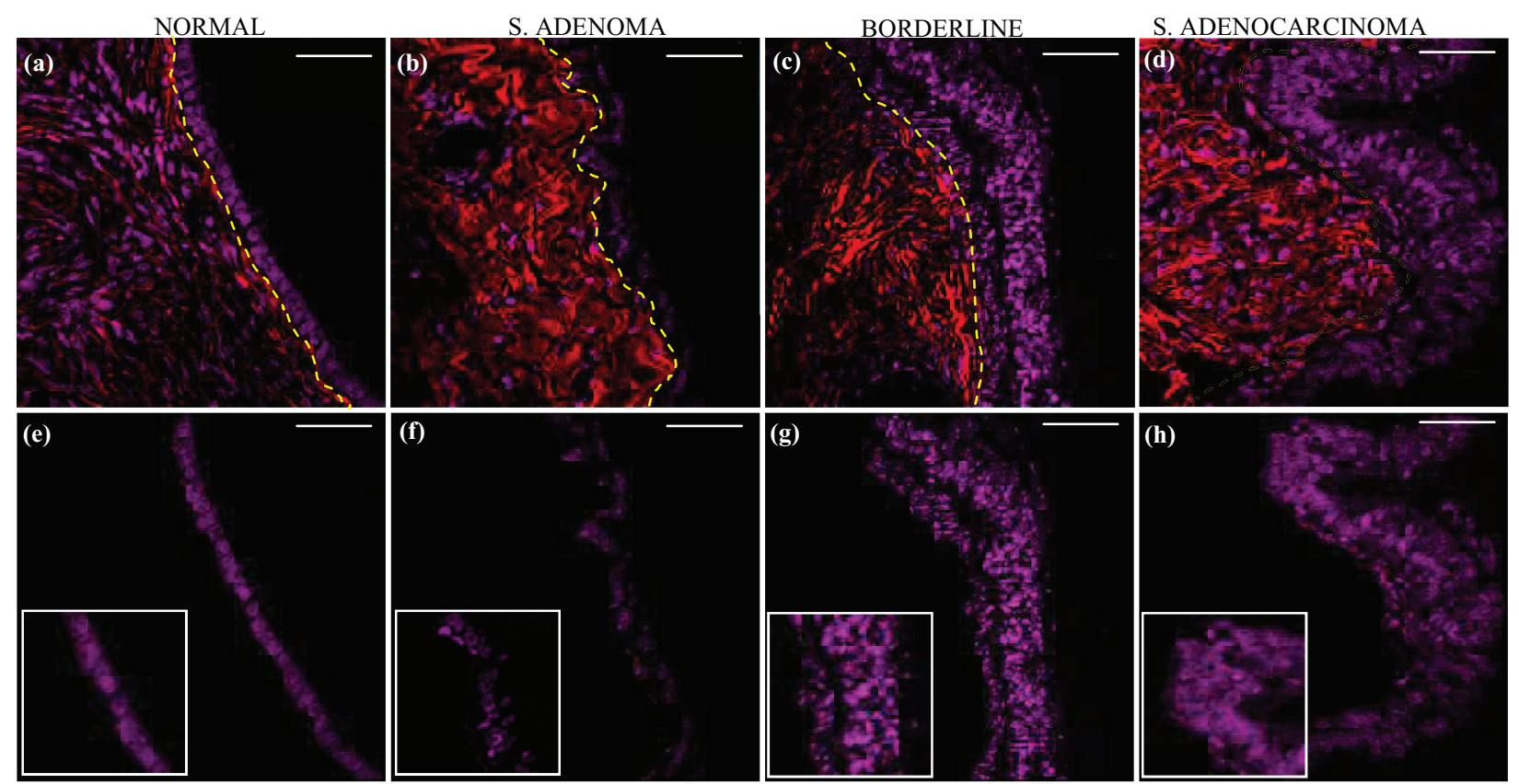

(i)

$* *$

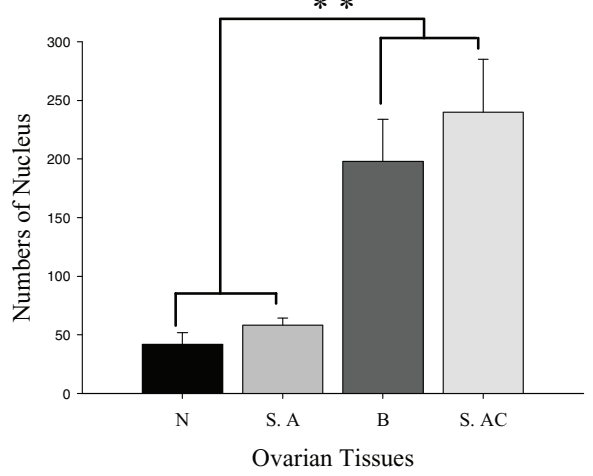

(j)

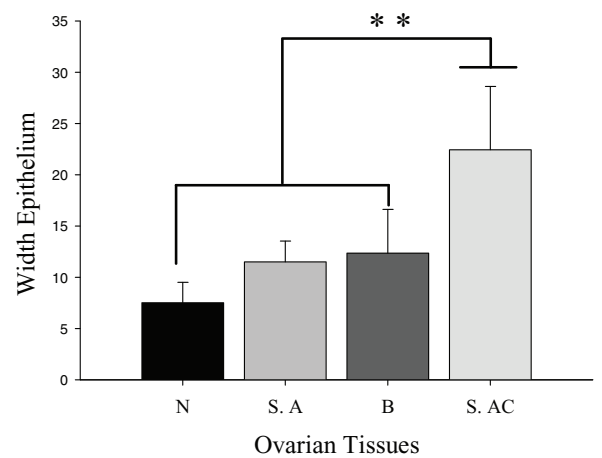

Fig. 4 Representative SHG + THG images $(512 \times 512$ pixels) at a two-photon excitation wavelength of $940 \mathrm{~nm}$ of tissues diagnosed as normal (a), serous adenoma (b), borderline (c), and serous adenocarcinoma (d). Epithelial/stromal interface is indicated (yellow outline). Quantitative analysis of epithelium for normal (e), serous adenoma (f), borderline (g), and serous adenocarcinoma (h) ovary samples, used to determine morphologic variables from THG signals: numbers of nuclei (i) and epithelial width (j). Inset shows more precisely the morphology of nuclei. Each bar represents the mean \pm S.D. of independent measurements. The total number of images from which these parameters were extracted was (normal: $n=5$, serous adenoma: $n=3$, borderline: $n=2$, serous adenocarcinoma: $n=10$ ) to calculated numbers of nuclei (i); and (normal: $n=15$, serous adenoma: $n=9$, borderline: $n=6$, serous adenocarcinoma: $n=30$; and includes the three regions for each image) to calculated epithelial width (j). N: normal, S.A: serous adenoma, B: borderline, S.AC: serous adenocarcinoma. Asterisks indicate a very significant $(* *, p<0.001)$ difference. All scale bars are $20 \mu \mathrm{m}$.

adenoma [Fig. 5(b)], serous borderline [Fig. 5(c)], and serous adenocarcinoma [Fig. 5(d)]. Examining visually the normal samples [Fig. 5(a)], we found that the collagen was more linearly structured with long, straight fibrils; whereas the collagen of abnormal samples exhibit a loss of fine structure and structural organization with wavy, collagen bands [Figs. 5(b), 5(c), and $5(\mathrm{~d})]$.

To quantitatively assess these collagen-related changes, we applied the Fourier transform analysis to the SHG images. The aim of the Fourier transform is to distinguish between the frequency components of an image. The intensities in our FFT images were compared and a representation of their periodicity within the image was given. This makes the Fourier transform a very good method of assigning a degree of organization to this image. In fact, for an image containing a set of aligned fibers, we expect a corresponding FFT image with higher values along the direction orthogonal to the direction of the fibers and its intensity plot is expected to show elliptic behavior. By contrast, for an image with randomly oriented fibers, the intensity plot of the corresponding FFT image should show circular behavior. The anisotropy of the image can be evaluated by measuring the ratio of the two axes of the ellipsis. ${ }^{28}$ The aspect ratio, ranging from 0 to 1, can give a measure of the anisotropy of the sample.

Collagen structure and density presented several differences in fibers alignment and disposition: in normal tissue, fibers were disposed in parallel to the major axis of the epithelia, while the serous adenoma showed visible and abundant dense fibers which followed the same axis orientation of the normal ovarian tissue. On the other hand, borderline and serous adenocarcinoma presented more irregular dense fibers [Figs. 5(a)-5(d)]. This means that the Fourier image of normal tissue and serous adenoma should be more elliptical than borderline and serous 

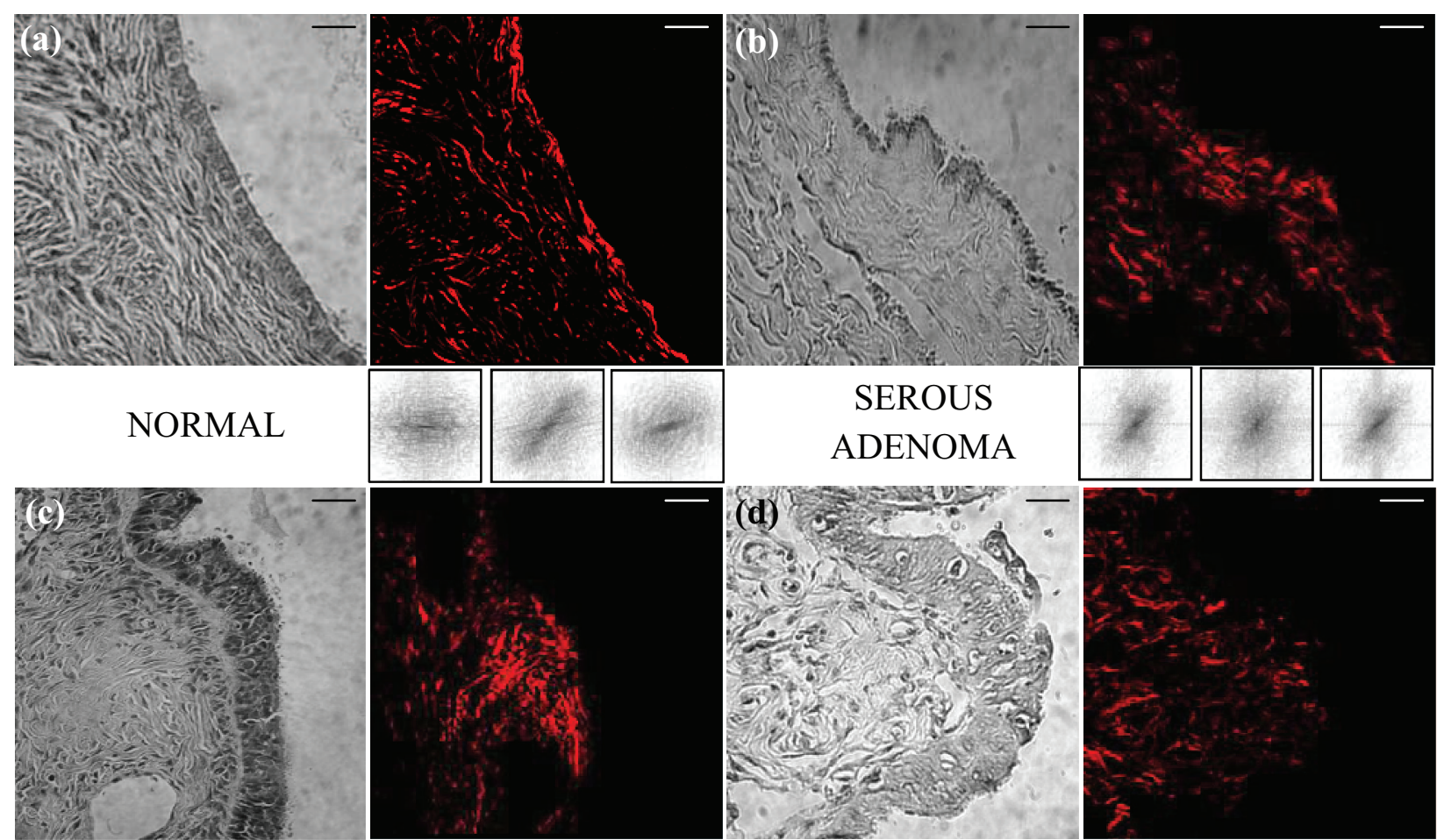

\section{SEROUS}

NORMAL

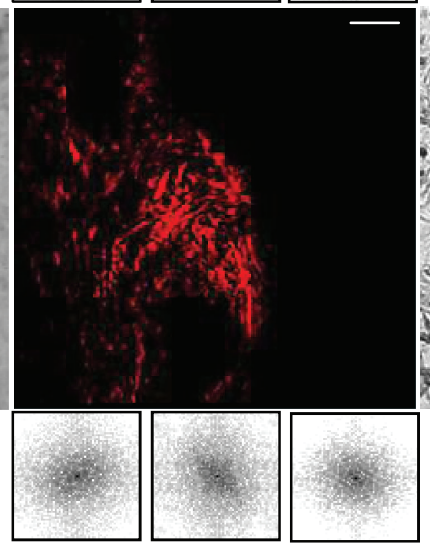

ADENOMA

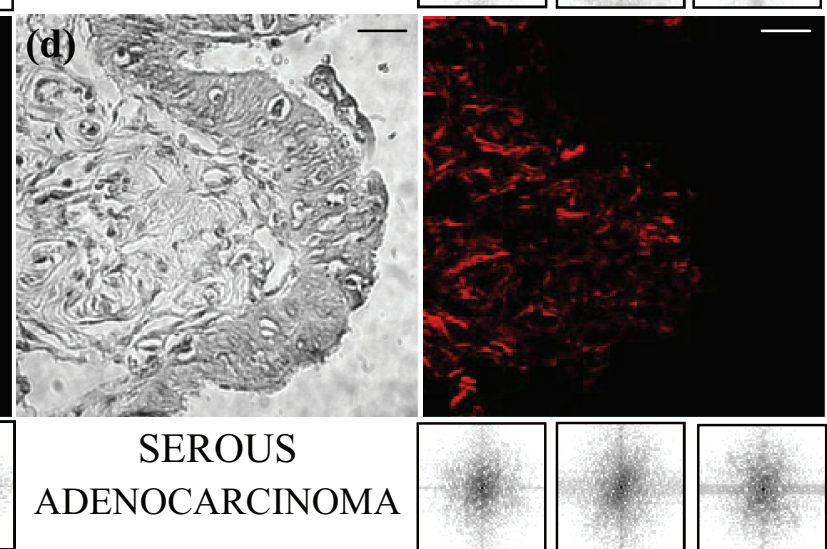

(e)

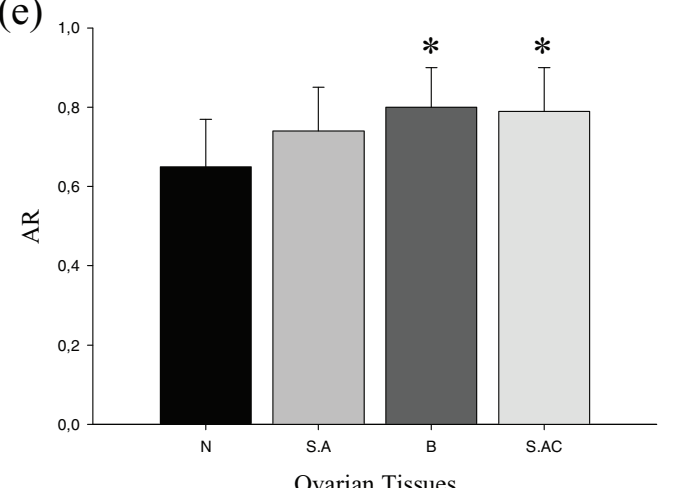

Fig. 5 Representative H\&E-stains and SHG images $(512 \times 512$ pixels $)$ at a two-photon excitation wavelength of $940 \mathrm{~nm}$ of tissues diagnosed as normal (a), serous adenoma (b), borderline (c), and serous adenocarcinoma (d). FFT intensity images obtained after 2D-DFT of each image are shown below SHG images. Results of the aspect ratio of ovarian samples averaged on all the examined samples (normal: $n=5$, serous adenoma: $n$ $=3$, borderline: $n=2$, serous adenocarcinoma: $n=10$ ) and on a three different regions (e). Each bar represents the mean $\pm S$.D. of independent 2D-DFT. N: normal, S.A: serous adenoma, B: borderline, S.AC: serous adenocarcinoma. Asterisks indicate a significant $(*, p<0.05)$ difference from normal samples. All scale bars are $20 \mu \mathrm{m}$. Ep: epithelium, St: stromal.

adenocarcinoma. The results obtained have confirmed what was expected: the FFT images of normal ovary and serous adenoma have shown a more elliptical profile [Figs. 5(a) and 5(b)] with respect to the FFT images of borderline and serous adenocarcinoma [Figs. 5(c) and 5(d)], which were found to have a more circular behavior. The AR value averaged on all the examined samples has shown a larger value for borderline $(0.80 \pm 0.10)$ and serous adenocarcinoma $(0.79 \pm 0.11)$ with respect to normal $(0.65 \pm 0.12)$, as was expected. These values were found to be statistically different at the 0.05 level after a two-sample statistical $t$-test [Fig. 5(e)]. Serous adenoma has an intermediate value
$(0.74 \pm 0.11)$. This result confirms the fact that normal ovary and serous adenoma are more organized tissues with respect to borderline and serous adenocarcinoma. Our data are consistent with previous studies that observed that the lower spatial frequencies increased, whereas the higher spatial frequencies decreased in the cancer group compared with the normal group, consistent with an alteration in collagen fibril fine structure (reduction of higher spatial frequencies) in the cancer group. ${ }^{15,16}$

To complete the analyses about collagen transformation in ovarian cancer, in Fig. 6 we quantified the collagen fiber angle relative to the epithelium. For this, we used SHG signals 

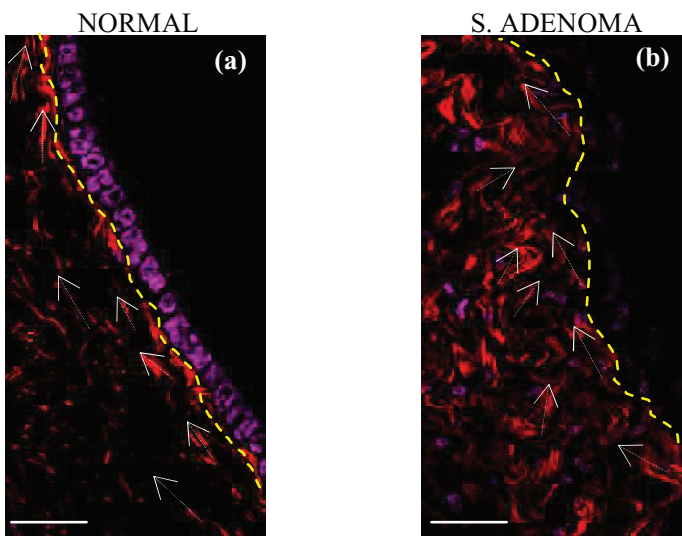

(e)

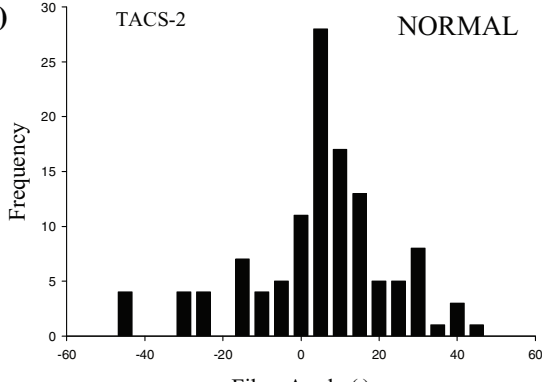

Fiber Angle ( ()

(g)
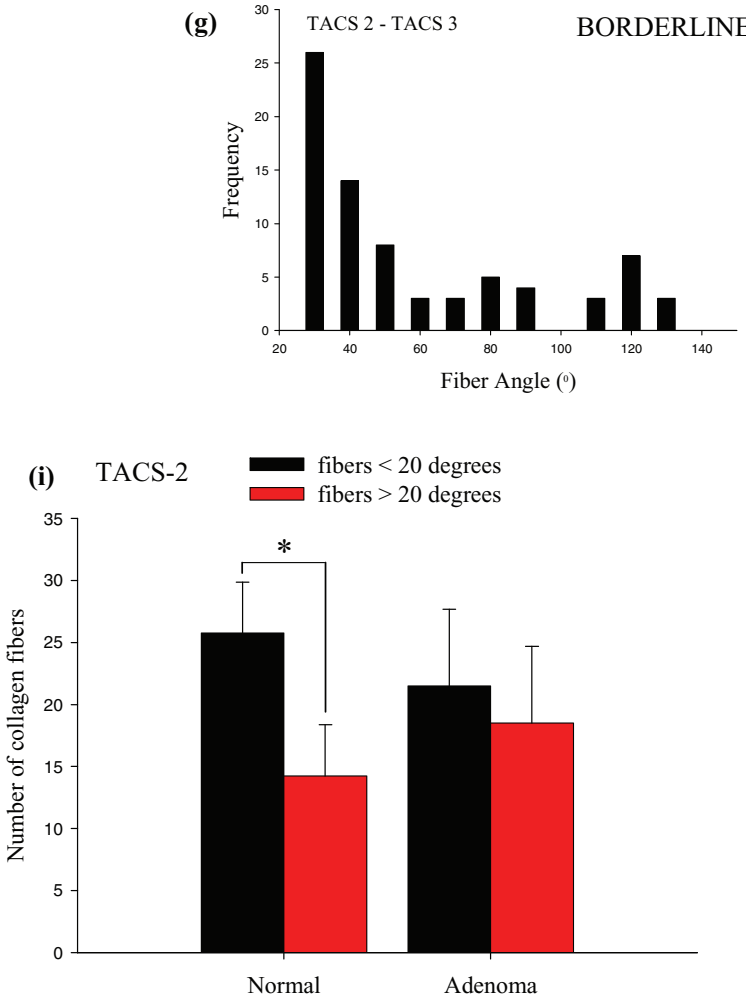
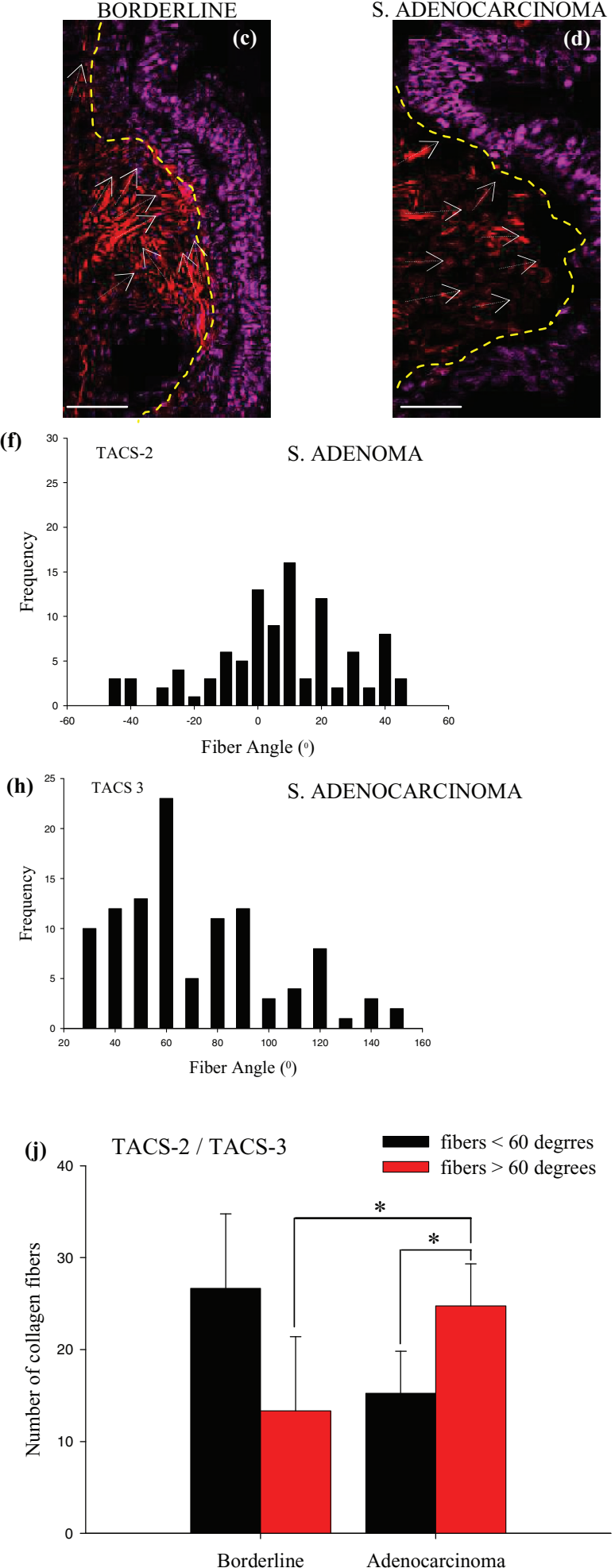

Fig. 6 Quantitative analysis of collagen fiber angles (white arrow) relative to the epithelial boundary (yellow outline) for normal (a), serous adenoma (b), borderline (c), and serous adenocarcinoma (d) ovary samples. Portions near the epithelial boundary were zoomed from Fig. 4 . TACS histograms from normal and serous adenomas displayed principally TACS-2 and are primarily noninvasive with 75\% (e) and 60\% (f) of their fibrils having an angle distribution around 0 deg (that is less than - 20 deg or more than $20 \mathrm{deg}$ ). In contrast, borderline (g) and serous adenocarcinoma (h) samples were different, possessing a broader fiber distribution and some regions of TACS-3 (distribution around 90 deg), with $25 \%$ and $60 \%$ of the fibrils distributed near $90 \mathrm{deg}$ (more than $60 \mathrm{deg}$ ), respectively. Values on the histogram were calculated from at least 160 fibers of tumor regions from at least 4 separate tumors. Statistical analysis from 40 fibers from each biopsy showed significant differences $(*, p<0.05)$. In normal samples (i), * indicates that the number of fibers with distribution $<20$ deg (TACS-2) was significantly greater. In serous adenocarcinoma samples (j), * indicates that the number of fibers with distribution $>60 \mathrm{deg}$ (TACS-3) was significantly greater. All scale bars are $20 \mu \mathrm{m}$. 
[(Figs. 6(a)-6(d)] and measured previously defined TACS (Ref. 14) [Figs. 6(e)-6(h)]. Specifically, we find the TACS-2, straightened (taut) collagen fibers stretched around the epithelium [Figs. 6(e) and 6(f)]; and TACS-3, identification of radially aligned collagen fibers that facilitate local invasion [Figs. 6(g) and $6(\mathrm{~h})]$. With TACS-3, a distribution of collagen fiber angles around 90 deg relative to the epithelium boundary would be indicative of high levels of local invasion while a distribution around $0 \mathrm{deg}$ would be associated with noninvasive regions of the epithelium. Clearly, our results show a gradual transformation of TACS-2 to TACS-3 as the stromal configuration passes from normal to an abnormal appearance. In normal ovary and serous adenoma, collagen fibers were principally distributed around 0 deg (see white arrows). In the first case, approximately $75 \%$ of them showed a distribution parallel to epithelium (angle $\leq$ $20 \mathrm{deg}$ ) [Fig. 6(d)] and in the second one $60 \%$ of the fibers show this behavior [Fig. 6(e)]. In contrast, borderline samples (with denser collagen fibrils) displayed more developed regions of TACS-2 (43\% fibers, $20 \mathrm{deg} \leq$ angle $\leq 40 \mathrm{deg}$ ) with more straightened fibers and some regions of TACS-3 (25\% fibers, $50 \mathrm{deg} \leq$ angle $\leq 110 \mathrm{deg}$ ) [Fig. 6(f)]. On the other hand, serous adenocarcinoma began to show regions of local invasion (TACS-3) with a peak of realignment fiber near $90 \mathrm{deg}$ (see white arrows) with respect to epithelium (60\% fibers, 50 deg $\leq$ angle $\leq 110 \mathrm{deg}$ ) [Fig. 6(h)]. In analyzing four representative images of each type of ovarian samples we quantified the number of fibers of collagen that are principally distributed around $0 \mathrm{deg}$ (angle $<20 \mathrm{deg}$ ) in normal and serous adenoma samples [Fig. 6(i)], and around $90 \mathrm{deg}$ (angle > $60 \mathrm{deg}$ ) in borderline and serous adenocarcinoma [Fig. 6(j)]. In normal and adenomas samples, the number of fibers with angle $<20$ deg was greater than fibers with angle $>20 \mathrm{deg}$. In the first case these values were found to be statistically different at the 0.05 level after a two-sample statistical $t$-test [Fig. 6(i)]. In borderline samples the number of fibers with an angle $<60 \mathrm{deg}$ was higher than fibers with an angle $>60 \mathrm{deg}$, so we cannot classify them as TACS-3. In contrast in serous adenocarcinoma samples the situation was the inverse, and these values were found to be statistically different. Furthermore, the number of fibers with an angle $>60 \mathrm{deg}$ is greater in adenocarcinoma than in borderline samples and statistically different, confirming the TACS-3 classification for adenocarcinoma tissues [Fig. 6(j)].

Our results showed that collagen was structurally modified (TACS-2 to TACS-3 transformation) above all in close proximity to focal areas of epithelial stratifications such as observed in serous adenocarcinoma samples. The interaction between the extracellular matrix and transformed neoplastic cells is thought to be a key element of cancer-induced angiogenesis and invasion. ${ }^{29}$ Recent research suggests that basement membrane loss occurs in surface epithelium from high-risk ovaries, ${ }^{13}$ perhaps promoting abnormal cellular function and directly affecting remodeling of the underlying extracellular matrix. Our results confirm this remodeling of extracellular matrix, being the first study to identify TACS-3 signatures in human ovarian tumors. In other studies the collagen alignment was used to quantify local invasion with the level of TACS-2 and TACS-3 in breast tumors and showed that the increased invasiveness is not only the result of earlier tumorigenesis that had more time to progress, but is also due to tumor cells that are fundamen- tally more invasive because they arose within collagen-dense tissues. $^{25}$

Taking all the results obtained from SHG signal together, we can say that in general our results are in line with the analyses recently presented by other authors. ${ }^{15-17}$ Briefly, Williams and co-workers, using thick, unstained samples, showed only qualitatively that collagen from human epithelial ovarian tumors was generally observed in thicker, less uniform bands than that from normal ovaries. ${ }^{16}$ Kirkpatrick and collaborators, working with 9 samples (normal $=4$ and cancer $=5$ ), demonstrated that the normal biopsies exhibit normotypic structured collagen fibrils near the epithelial surface and the biopsies from patients with cancer exhibit a loss of fine structure and structural organization with wavy, collagen bands. ${ }^{15}$ The work of Nadiarnykh and collaborators performed on a few tumors $(n=3)$; demonstrated that malignant ovaries were characterized by denser collagen such as we saw. However, they also showed that malignant ovaries present lower cell density, as well as higher regularity at both the fibril and fiber levels. ${ }^{17}$ Nevertheless, it is difficult to make a proper comparison between our study and the previous two, since they do not classify the type of tumor studied. For example, interpretations of Nadiarnykh do not coincide with our observations in borderline and serous adenocarcinoma, but are very similar to our observations in serous adenoma type ovarian.

In particular, some difference can be found between our study and those presented in the previous paragraph. The more uniform pattern of wavy collagen found by others probably arises because of differences in age of the patients evaluated. In older patients such as our cases, the collagen fibrils normally seem more diffuse. ${ }^{15}$ As well as the biopsy conditions, old stored samples could have suffered some degradation if compared with observations of fresh samples. Another important difference between the reports is that we used standard H\&E histopathology cross sections (4- $\mu \mathrm{m}$ thick) and the other authors used en face optical sections from thicker tissue slices (100 to $200 \mu \mathrm{m}$ ); thus, depending on the depth at which the observation is made the structure of collagen fibers may be different. In general, with increased depth, the collagen is both depleted and lacks integrity. Additionally, thick tissue imaging requires signal collection in the backward direction. Due to the vastly different features of forward and backward images, it is essential to understand backscattering of forward components for SHG in vivo and thick tissue imaging. ${ }^{30}$

In summary, taking our results together we can see collagen transformation (difficult to see in real time short experiment) and we can say that collagen presentation in ovarian normal and tumoral tissues obeys specific patterns (not visible in standard H\&E-stained sections) which are possible to detect from SHG signals.

\section{Conclusion}

We demonstrated that nonlinear microscopy techniques could be used to perform a retrospective study in a long time scale if it is possible to find biopsies of the same patient at different moments. Unfortunately, silent ovarian cancer is not ideal for this because it is not a disease followed with biopsies over time. Using a stored library of H\&E stained samples of human ovarian cancer and combining the TPEF-SHG-THG imaging approach, we obtained complementary information about the 
epithelium/stroma interface, such as the transformation of epithelium surface (THG) and the overall fibrillar tissue architecture (SHG). In this work we also demonstrated that multicontrast nonlinear microscopy can differentiate between cancerous and healthy tissue. More specifically, we can effectively make a distinction between normal, benign tumor, borderline, and malignant specimens according to their collagen disposition and compression levels within the extracellular matrix, as well as the dimensions of the respective epithelia layers.

These results suggest that, with our viable imaging system, we can qualitatively and quantitatively assess endogenous optical biomarkers of the ovary with THG and SHG microscopy. Our data demonstrate that optical techniques are useful adjuvants to detect pathological changes associated with ovarian cancer. Given the morphological information present in multiphoton imaging of the ovary, we are planning in the future to translate this analysis to in vivo and three-dimensional studies.

\section{Acknowledgments}

The authors are grateful to FAPESP. This work is also linked with CEPOF (the Optics and Photonics Research Center, FAPESP) and the National Institute of Photonics Applied to Cell Biology (INFABIC).

\section{References}

1. W. R. Zipfel, R. M. Williams, and W. W. Webb, "Nonlinear magic: multiphoton microscopy in the biosciences," Nat. Biotechnol. 21 (11), 1369-1377 (2003).

2. H. Chen, H. Wang, M. N. Slipchenko, Y. K. Jung, Y. Shi, J. Zhu, K. K. Buhman, and J. Cheng, "A multimodal platform for nonlinear optical microscopy and microspectroscopy," Opt. Express. 17 (3), 1282-1290 (2009).

3. M. Mathew, S. I. C. O. Santos, D. Zalvidea, and P. Loza-Alvarez, "Multimodal optical workstation for simultaneous linear, nonlinear microscopy and nanomanipulation: Upgrading a commercial confocal inverted microscope," Rev. Sci. Instrum. 80(7), 1-11 (2009).

4. P. P. Provenzano, K. W. Eliceiri, and P. J. Keely, "Multiphoton microscopy and fluorescence lifetime imaging microscopy (FLIM) to monitor metastasis and the tumor microenviroment," Clin. Exp. Metastasis 26(4), 357-370 (2009).

5. P. P. Provenzano, C. T. Rueden, S. M. Trier, L. Yan, S. M. Ponik, D. R. Inman, P. J. Keely, and K. W. Eliceiri, "Nonlinear optical imaging and spectral-lifetime computacional analysis of endogenous and exogenous fluorophores in breast cancer," J. Biomed. Opt. 13(3), 031220 (2008).

6. P. P Provenzano, K. W. Eliceiri, J. M. Campbell, D. R. Inman, J. G. White, and P. J. Keely, "Collagen reorganization at the tumor-stromal interface facilitates local invasion," BMC Med. 4(1), 1-16 (2006).

7. S. Hooper, J. F. Marshall, and E. Sahai, "Tumor cell migration in three dimensions," Methods Enzymol. 406, 625-643 (2006).

8. E. Sahai, J. Wyckoff, U. Philippar, J. E. Segall, F. Gertler, and J. Condeelis, "Simultaneous imaging of GFP, CFP and collagen in tumors in vivo using multiphoton microscopy," BMC Biotechnol. 5(14), 1-9 (2005).

9. D. Débarre, W. Supatto, A. M. Pena, A. Fabre, T. Tordjmann, L. Combettes, M. C. Schanne-Klein, and E. Beaurepaire, "Imaging lipid bodies in cells and tissues using third-harmonic generation microscopy," Nat. Methods 3(1), 47-53 (2006).

10. N. Elie, B. Plancoulaine, J. P. Signolle, and P. Herlin, "A simple way of quantifying immunostained cell nuclei on the whole histologic section," Cytometry, Part A 1(56), 37-45 (2003).

11. A. Tuer, D. Tokarz, N. Prent, R. Cisek, J. Alami, D. J. Dumont, L. Bakueva, J. Rowlands, and V. Barzda, "Nonlinear multicontrast microscopy of hematoxylin-and-eosin-stained histological sections," J. Biomed. Opt. 15(2), 026018 (2010).
12. U.S. Cancer Statistics Working Group, United States cancer statistics: 2003 incidence and mortality, Atlanta: US Department of Health and Human Services, Centers for Disease Control and Prevention and National Cancer Institute (2006).

13. I. H. Roland, W. L. Yang, D. H. Yang, M. B. Daly, R. F. Ozols, T. C Hamilton, H. T. Lynch, A. K. Godwin, and X. X. Xu, "Loss of surface and cyst epithelial basement membranes and preneoplastic morphologic changes in prophylactic oophorectomies," Cancer 98(12), 2607-2623 (2003).

14. S. Bhoola and W. J. Hoskins, "Diagnosis and management of epithelial ovarian cancer," Obstet. Gynecol. 107(6), 1399-1410 (2006).

15. N. D. Kirkpatrick, M. A. Brewer, and U. Utzinger, "Endogenous optical biomarkers of ovarian cancer evaluated with multiphoton microscopy," Cancer Epidemiol. Biomarkers Prev. 16(10), 2048-2057 (2007).

16. R. M. Williams, A. Flesken-Nikitin, L. H. Ellenson, D. C. Connolly, T. C. Hamilton, A. Y. Nikitin, and W. R. Zipfel, "Strategies for highresolution imaging of epithelial ovarian cancer by laparoscopic nonlinear microscopy," Transl. Oncol. 3(3), 181-194 (2010).

17. O. Nadiarnykh, R. B. LaComb, M. A. Brewer, and P. J. Campagnola, "Alterations of the extracellular matrix in ovarian cancer studied by second harmonic generation imaging microscopy," BMC Cancer 10(94), 1-14 (2010).

18. R. E. Scully, R. H. Young, P. B. Clement, Eds., Atlas of Tumor Pathology: Tumors of the Ovary, Maldeveloped Gonads, Fallopian Tubes and Broad Ligament, 3rd Ed., Armed Forces Institute of Pathology, Washington, D.C. (1998).

19. A. Tuer, L. Bakueva, R. Cisek, J. Alami, D. J. Dumont, J. Rowlands, and V. Barzda, "Enhancement of third-harmonic contrast with harmonophores in multimodal non-linear microscopy of histological sections," Proc. SPIE 6860, 686005 (2008).

20. C. Yu, S. Tai, C. Kung, W. Lee, Y. Chan, H. Liu, J. Lyu, and C. Sun, "Molecular third-harmonic-generation microscopy through resonance enhancement with absorbing dye," Opt. Lett. 33(4), 387-389 (2008).

21. M. W. Conklin, P. P. Provenzano, K. W. Eliceiri, R. Sullivan, and P. J. Keely, "Fluorescence lifetime imaging of endogenous fluorophores in histopathology sections reveals differences between normal and tumor epithelium in carcinoma in situ of the breast," Cell. Biochem. Biophys. 53(3), 145-157 (2009).

22. P. J. Campagnola, A. C. Millard, M. Terasaki, P. E. Hoppe, C. J. Malone, and W. A. Mohler, "Three-dimensional high-resolution secondharmonic generation imaging of endogenous structural proteins in biological tissues," Biophys. J. 82(1), 493-508 (2002).

23. S. J. Lin, S. H. Jee, C. J. Kuo, R. J. Wu, W. C. Lin, J. S. Chen, Y. H. Liao, C. J. Hsu, T. F. Tsai, Y. F. Chen, and C. Y. Dong, "Discrimination of basal cell carcinoma from normal dermal stroma by quantitative multiphoton imaging," Opt. Lett. 31(18), 2756-2758 (2006).

24. R. Cicchi, S. Sestini, V. De Giorgi, P. Carli, D. Massi, and F. S. Pavone, "Basal cell carcinoma imaging and characterization by multiple nonlinear microscopy techniques,” Biophys. J. Supplement: S, 157A (2007).

25. P. P. Provenzano, D. R. Inman, K. W. Eliceiri, J. G. Knittel, L. Yan, C. T. Rueden, J. G. White, and P. J. Keely, "Collagen density promotes mammary tumor initiation and progression," BMC Med. 6(11), 1-15 (2008).

26. E. Brown, T. McKee, E. di Tomaso, A. Pluen, B. Seed, Y. Boucher, and R. K. Jain, "Dynamic imaging of collagen and its modulation in tumors in vivo using second-harmonic generation," Nat. Med. 9(6), 796-800 (2003).

27. O. Nadiarnykh and P. J. Campagnola, "Retention of polarization signatures in SHG microscopy of scattering tissues through optical clearing," Opt. Express 17(7), 5794-5806 (2009).

28. P. Matteini, F. Ratto, F. Rossi, R. Cicchi, C. Stringari, D. Kapsokalyvas, F. S. Pavone, and R. Pini, "Photothermally-induced disordered patterns of corneal collagen revealed by SHG imaging," Opt. Express 17(6), 4868-4878 (2009).

29. G. Kurban, V. Hudon, E. Duplan, M. Ohh, and A. Pause, "Characterization of a von Hippel Lindau pathway involved in extracellular matrix remodeling, cell invasion, and angiogenesis," Cancer Res. 66(3), 13131319 (2006).

30. F. Légaré, C. Pfeffer, and B. R. Olsen, "The role of backscattering in SHG tissue imaging," Biophys. J. 93(1), 1312-1320 (2007). 